\title{
Guest Reviewers 1988
}

The Editorial Board acknowledyes with thanks the assistance of the following guest reviewers.

Abou-Madi, M. / MONTREAL

Ananthanarayan, C. / TORON To

Anhalt, Edward F. / WINNIPEG

Artru, Alan A. / SEattle, Wa

Bain, J.A. / LONDON

Bedder, Marshall D. / PORTLAND, OR

Berman, Lawrence S. / GAINESvitLL, FL

Bevan, J.C. / MONTREAL

Biehl, Diane / WINNIPEg

Blane, Victor Faria / MONTREAL

Bond, D.M. / LONDON

Bose, D. / WINNIPEG

Bristow, G. / WINNIPEG

Britt, Beverley A. / TORON To

Bryant, D. / ST. JOHN's

Burrows, Frederick A. / TORONTo

Cannon, John E. / WINNIPEG

Carle, H. / edmonton

Cattran, C. / OTTAWA

Chung, Frances / TORONTo

Comm, Glenn / EDMONTON

Covino, Benjamin G. / Boston, MA

Cruchley, Patricia / TORONTO

Cucchiara, Roy F. / RocHESTER, MN

Donati, François / MONTREAL

Donen, Neil / WINNIPEG

Douglas, M. Joanne / vancouver

Duke, Peter C. / WINNIPEG

Eagle, C.E. / CaLGary

Edelist, Gerald / IORONTO

Eger, E.I. II / San francisco, CA

Enright, Angela / SASKa TOON

Fear, David W. / TORON'To

Finegan, B. / EDMONTON
Fisher, J.A. / TORONTO

Froese, Alison B. / KInGston

Gambling, David R. / VANCOUVER

Gelb, Adrian / LONDON

Ghignone, Marco / PITTSBlRGH, PA

Greengrass, Roy / WINNIPEG

Hall, Richard I HALIFAX

Hamilton, R.C. / CALGARY

Hardy, Jean-François / MONTRÉAL

Herrick, Ian A. I KINGSTON

Hewson, J.R. I HAMILTON

Holaday, Duncan A. / NASHVILLE, TN

Hosking, Denis A. / WINNIPEG

Hudson, Robert J. / WINNIPEG

Imrie, David D. / HALIFAX

James, M.F.M. / JOHANNESBLRG, SOUTH AFRICA

Jenkins, L.C. I VANCOUVER

Kambam, J.R. / NASHVILLE, TN

Kaufman, S. / EDMONTON

Kehler, Chris H. / WINNIPEG

Knill, R.L. / LONDON

Koch, Jean-Paul / TORONTO

LaBella, Frank S. i WINNIPEG

Laws, Alan K. / TORonto

Lee, Kwok C. / TORONTO

Lerman, Jerrold / TORONTO

Lewis, G.W. I OTTAWA

Limoges, Pietre / MONTREAL

Longmuir, John / WINNIPEG

Lyin, Anne / SEattle, Wa

Mallampati, S. Rao / BOSTON, MA

Maltby, Roger / CALGary

Manninen, Pirjo / LONDON

Manson, H.J. / ST. JOHN's 
Mass, Michael / WINNIPEG

McCullough, D.W. / WINNIPEG

McIntyre, J.W.R. / EDMONTON

McKenna, Jocelyne / OTTAwA

McMorland, G.H. / vancouver

Mezon, B.J. / LONDON

Miller, Ronald D. / SAN FRANCISCo, CA

Moote, C.A. / London

Morison, D.H. / HAMILTON

Murkin, J.M. / LONDON

Murphy, Michael / HALIFAX

Mutch, W.A.C. / WINNIPEG

Naguib, Mohamed/RIYADH, SAUDI ARABIA

Nathan, H. / OTTAwA

Nielson, Warren / LONDON

Nicolle, L.E. / WINNIPEG

Nolan, Kevin / OTTAWA

Ong, B. Y. I WINNIPEG

Oxorn, Donald C. / HaLIFAX

Pash, Michael P. / CaLGary

Penny, Foster J. / LONDON

Perera, E. I TORONTo

Pope, W.D.B. / WINNIPEG

Poppers, Paul J. / stony brook, NY

Pounder, David / hallFax

Ravussin, Patrick A. / Lausanne, switzerland

Reimer, D.H. / WINNIPEG

Rhine, Elliot J. / OTTAWA
Robblee, Jarnes A. / OTtawa

Robertson, K.M. / LONDON

Rogers, Kent H. / TORONTO

Rose, Keith / TORONTO

Roy, W. Lawrence / TORONTO

Samson, Benoit : OTTAWA

Samulska, H. I TORONTo

Sandler, Alan N. / TORONTO

Seargeant, Lorne / WINNIPEG

Seshia, S. / WINNIPEG

Sessler, Daniel I. i SAN FRANCISCO, CA

Slinger, P. / MONTRÉaL

Sloan, I.A. / TORONIO

Smith, J. Bruce i MONTREAL

Sosis, Mitchell / Indianapolis, IN

Steward, David I VANCOUVER

Stoelting, Robert K. / INDIANAPOLIS, IN

Teasdale, S. / TORONTO

Trop, Davy / MONTRtél

Ullyot, Suzanne i WINNIPEG

Vitez, Terry $S$. / LAS VEGAS, NV

Wahba, W.M. / MONIREAL

Weeks, Sally K. / MONTREAL

Wexler, H.R. / LaNdoN

Whalley, Davic G. / MONTRÉAL

White, Ian / WINNIPEG

Williams, R. Tudor / CALGARY 